\title{
Improvement of construction of combined double-circular hoe boot
}

\author{
V. Bulgakov, \\ Academician of the National Academy of Sciences of Ukraine, Doctor of Technical Sciences, \\ National University of Bioresources and Natural Resources of Ukraine \\ V. Gorobey, \\ Candidate of Technical Sciences, NPO "Selta" NSC "Institute of Mechanization and \\ Electrification of Agriculture"
}

The purpose. To develop the design of boot consolidating positive performances of double-circular and hoe boots, working on coarsely prepared with plant residues soils and stably ensuring depth of seeding on the heightened speeds of sowing. Methods. Mathematical simulation, experimental and statistical. Probes of the combined double-circular hoe boots at sowing by selection-seed-growing seeder $\mathrm{SN}-16$ in the assembly unit with tractor T-25 are carried out at seeding millet in field seed-fallow row-crop rotation on dark-chestnut medium-loamy soil. Results. Parameters of the combined double-circular hoe boot with the improved gear of regulation of depth of drilling of seeds are justified. On the basis of the equivalent main circuit of construction of the boot necessity of application of spring of the set rigidness for provision of stability of seeding seeds on the set depth within the limits of agrotechological the tolerance is justified. Dependences for calculation of rational spring rate which consider geometrical parameters of construction of the boot are gained. Probes in field conditions confirm advantages of new designs. Conclusions. The seeder with the combined double-circular hoe boot stacks seeds on solid seeds bed, eliminates lag of removal of seeds for limits of agrotechological tolerance, including on the heightened speeds of sowing and covering rollers which are fixed with an opportunity of regulation of the position. That is advantages of boots with new designs.

Key words: combined boot, construction, seeding, scheme, parameters, account, spring, pressure, agrotechological the tolerance.

Formulation of the problem. It is widely known that the quality of sowing of cereals significantly affects the yield of high yields. In this case, the correct selection of the combine group of the grain drill can guarantee the uniform distribution of seeds to the required depth of its wrapping in the soil. For seeding of grain crops in production conditions and in breeding and sowing business, two-sowing seeders are used which do not ensure the stability of seeding in depth, especially at high seed rates. Nalalnikovye coulters more evenly stack seeds at a given depth, on the formed solid seedbed, but their use is not widespread, because they, unlike the disk, require thorough pre-planting soil preparation. Technical solutions for the construction of coulter planes, which ensure the hanging of seeds of cereals in mulched plant residues of soil at a stable depth, have not yet been found so far wide practical application due to technological imperfections and manufacturing difficulties. Therefore, the improvement of the design of the disk-anchor coil unit and the substantiation of its structural and kinematic parameters is an actual scientific and technical task..

According to the agronomic requirements, seed sown should be placed in the soil at a strictly defined depth [9]. Accepted depth of wrapping of wheat, rye, oats, barley on light soils is $4.5-6.0 \mathrm{~cm}$, on medium wet soils $2.5-4.5 \mathrm{~cm}$, heavy $-2-4$, in the zone of wind erosion $-6-8 \mathrm{~cm}$. The number of seed sown to a given depth must be at least $80 \%$ [3]. The correct selection of a coarse group in a grain drill can guarantee the seed placement at the desired depth at the crop.

Analysis of recent publications. Two-disc coulter [16], which is usually secured to a seeder frame on an elastic radial suspension [13], is the most universal coulter for grain crops. Among their advantages is the possibility of sowing on roughly treated lumpy soil with plant residues. In this case, the two-disc coulter effectively cut such a soil and even roll through obstacles. Bimetallic coils are widely used in traditional and, so-called, minimal tillage technologies. They are also used in the drainage phases of 
ordinary seeders of the model range of SZ (produced by OJSC "Chervona zirka") [8]. It should be emphasized that the company John Deere produces grain seeders $730,455,740 \mathrm{~A}$ with two-disc coulter, which have a chamfer on the discs and the arrangement of shoots in chess. Double-bore drills are installed on Lemken's Solitair grain pneumatic seed drills for work on floating and mulch fronts, Great Plains grain and seed drills HT and SRN are equipped with two-disc coulters for direct sowing [4].

In selective seed seeders such as SN-16, SCS-6A, CH-10C (K) [12] and in their modern modifications most often use two-disc coulter. The drawbacks of this type of coulter consist in the fact that they do not satisfy the agronomic requirements for the compacted bottom of the seeding furrow and, as a result, do not provide the seeded seed, the required humidity regime at the chosen depth in the soil [7]. In addition, experiments conducted at VISGOM and VIM have proved that these coulters also unevenly distribute the seeds in depth in the furrow. It is established that the speed of the forward movement of the drill more than $8 \mathrm{~km} / \mathrm{h}$ a significant amount of seeds even carried out on the surface of the field, and at a given depth, only $38-56 \%$ of the seed remains. With deviations in the depth of seeding by $18 \mathrm{~mm}$, their field germination decreases and amounts to approximately $54 \%$ [15].

Ropes of the spit type most naturally form the furrow and seedbed, provide the appropriate contact of the seed with the soil, withstand the required depth of seeding. They are also more convenient because the quality of their work is less dependent on the speed of the drill and they do not drag the plant residues into the crop rotation, in addition, they effectively shield the plant remains on the sides $[14,17]$. But while the coils of this type require careful preparation of the soil and very sensitive to its physical and mechanical state.

Known works on the improvement of the designs of disk coulter [1, 2, 15], as well as on the development of its simplified design with the combination of advantages of two-discus and anchor types of coils $[5,10]$, technical solutions of which have not been widely used in industrial practice and an experimental business due to the complexity of manufacturing and regulation, as well as known disadvantages, are characteristic, in particular, for two-disc coulter.

Therefore among the priority tasks of breeding crops is the creation of a design of a cultivator for seeding of grain crops, which ensures an increase in the stability of the depth of seeding and seed distribution along the bottom of the seed furrow by means of the elimination of the contact of the seeds with the inner surface of the rotating discs when driving the coulter.

The purpose of the research is to develop technical and theoretical decisions for the design of the coil, which combines the positive characteristics of the two-disc and coil-type anchor-type, working on roughly prepared, with plant residues of soils, and consistently provides depth of wrapping in the soil of the seed at higher seeding speeds..

Research methodology. Proceeding from the conditions for ensuring the stability of the depth of seeding of grain crops, determining the parameters of a combined two-disk anchor coil, for a mathematical description of its movement in the soil, the scheme of forces acting on it during the movement, without taking into account the forces of inertia, is analyzed. A constructive and equivalent circuit and a method for calculating the stiffness of the spring have been developed to ensure maximum deviation of the seeding depth within the limits of agrotechnical tolerance.

The results of calculations of the design parameters of the coil of an improved design with given geometric parameters, obtained on a personal computer in the Mathcad.

Investigation of experimental specimens was carried out in field crop rotation on dark chestnut medium-sandy soils. Millet in crop rotation was placed after winter wheat, the main work was plowing to a depth of $20-22 \mathrm{~cm}$. In this preplant soil cultivation consisted of harrowing conducted in the spring, and at the optimal seeding time, pre-sowing cultivation was carried out at the recommended depth of seeding of 3 to $5 \mathrm{~cm}$ The seed was started immediately after the cultivation was completed. Agrotechnological research was carried out in field conditions with a sowing machine $\mathrm{CH}-16$ in an aggregate with a tractor T-25. The drill is equipped with 13 coils, of which: 7 pcs. - combined double-disk and anchor and 6 pcs. Basic dual disks.

The comparative definition of the depth of wrapping of millet seeds was carried out directly with the use of coulterers of different designs and assess their impact on the uniformity of obtaining stairs and the development of plants. 
The assessment of the quality of the sowing (depth of wrapping, the uniform distribution of the seed), depending on the speed of the sowing unit and the type of the stem, was carried out at different speeds of the grain selection seeder: $7.2 ; 9.6$ and $14.5 \mathrm{~km} / \mathrm{h}$.

Research results. The basis of the modernization of the cultivator was the task of improving the design of a combined two-disc coulter equipped with a pin-like part hingedly attached between the discs to the coulter's body, which would increase the uniformity of the depth of seeding by way of eliminating the lifting of the lower part of the nursery to a height that exceeds the size of agrotechnological tolerance. The given task is solved due to the fact that in the design of the coil, which contains the installed, with the possibility of rotation of the angle forward in the course of the coil, two flat disks and the coil body is located between them, and between the disks with the help of a pivot suspension and a spring to his body is attached to a pile a part that holds the bell and hoe. The installation of a bevel-like part of the coil between the disks by means of fastening it to the body with the help of a hinged suspension and a spring makes it possible for the nailer, a pin-shaped part of the coulter to move along the prepared furrow, previously opened discs, sealing the bottom of the seed groove, with the seed not touching the rotating surfaces of the discs eliminates the transfer of seeds from the soil to the upper part of the furrow, increasing the stability of the depth of seeding and seeding in the soil.

The combination of the advantages of two-disc and pin-shaped coils is realized in the design of a combined coil [11]. In the diagram (fig. 1) of the combined two-disk anchor coil, the drawings are shown: a - side view when the left disk is removed; $b$ - cross section in A-A in the enlarged form.

The coulter consists of a housing 1, in which two axle disks are mounted on the axes 2 with the possibility of rotation forward in the direction of the combuster. 3. Between the disks with the housing is a holder 4 , consisting of two cheeks 5 . The void created by the cheeks, a rolled part of a combination coil, which has a bell 6 and a native plunger 7 , and is attached to the housing 1 with a spring 8 , and a cheek holder with a pivot suspension 9, which is located in relation to the coulter's stroke, behind the globular part. In addition, the design of a new type of coulter involves rolling rollers (not shown in the diagram in Fig. 1).

The combined double-disc anchor coil works in the following way. When driving the coulter in the soil, the discs 3 , rotating on the axles, open the seed groove. The space 4 of the holder 4 , created by the cheeks 5 , is suspended with the help of the pivot suspension 9 , and the pile-like part of the coil spring 8 is pressed by the lower part of the feeder 7 to the bottom of the seed groove and, accordingly, compresses it.

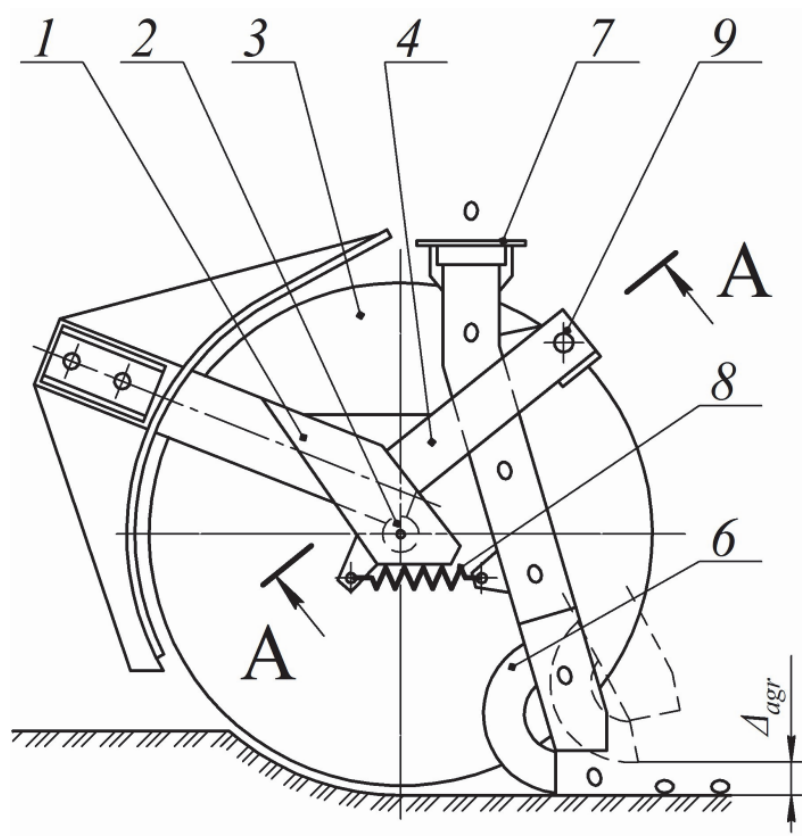

a

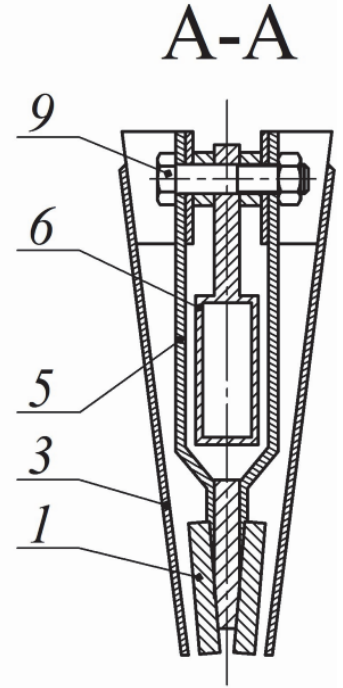

$b$

1 - body; 2 - axle; 3 - disk; 4 - holder; 5 - cheek; 6 - bell; 7 - feeder; 8 - a spring; 9 - hinged suspension

Fig. 1. Scheme of a combined two-disk anchor coil: a - side view when removed from the left disk; $b$ section by $A-A$ 
For a theoretical study of the motion in the soil of a new, combined two-disk anchor coulter, in order to determine its optimal structural and kinematic parameters that ensure the stability of the seeding depth, an equivalent scheme should be made, in which the forces acting on it during movement should be shown. Since the relative movements of the rougher part of the coil in the soil relative to the discs occur too slowly, then all grounds are neglected by the forces of inertia. Therefore, in the first approximation, we will consider the motion of a new coulter in the soil, as the motion of a balanced system in accordance with the equivalent scheme given in Fig. 2

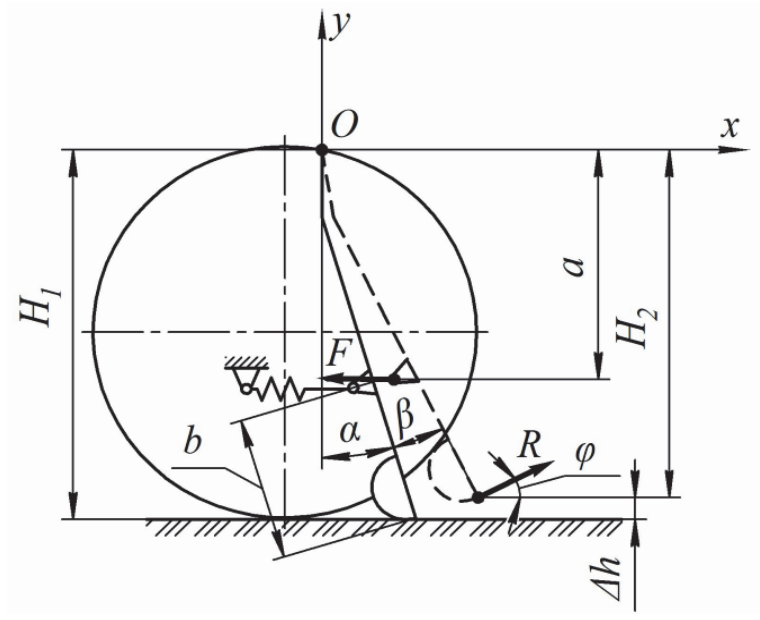

Fig. 2. Equivalent circuit of a combined two-disk anchor coil

During the movement in the soil on the rolled part of the coulter there are two forces: $R$ - the strength of the ground resistance and $F$ - the strength of the spring elasticity

We will also show the main dimensional characteristics of the elements of the improved design of the coil: $H_{1}$ - distance from the axis of the hinged suspension to the bottom of the seed furrow; $H_{2}$ - seeding depth at maximum deviation; , $a, b$ - design parameters of the coil; $\alpha$ - angle of deflection of a ridged part; $\beta$ - angle of deviation of the seed movement; $\varphi$ - angle of deviation of the direction of force of ground resistance from the horizontal; $\Delta h$ - change in the depth of seeding.

The hinged suspension 9, in accordance with the improved design (Fig. 1, a), is attached to the carrier 3 connected to the housing 1 of the holder 4 , consisting of two cheeks 5 , which create a space in which a pivot part is installed in such a way that the axis of the pivot suspension a rope-shaped part is located behind it during the course of the coulter, and the projection of the axis on the bottom of the seed groove coincides with the contact area of the lower part of the nurl9 and the bottom of the seed furrow. At this distance from the axis of the pivot suspension to the bottom of the seed groove $H_{1}$ can be determined from such a ratio:

$$
H_{1}\left(1-\cos \beta_{\max }\right)=\Delta h<\Delta_{\text {agr. }},
$$

where $\beta_{\max }$ - maximum deviation angle of the pivot part from the axis of the pivot suspension; $\Delta_{\text {agr. }}-$ the value of the agrotechnical tolerance on the size of the bookmark of the seed.

If we assume that during a stationary motion this system is in a condition of equilibrium, then for it it is possible to make such a condition of equilibrium:

$$
\sum_{k=1}^{n} M_{o}\left(\bar{P}_{k}\right)=0
$$

where $M_{o}$ - moments of acting forces $\bar{P}$ relative to the point $O$ hinged pendant (fig. 2).

On the basis of expression (2) we write the equation of equilibrium at the maximum deviation of the sowing:

$$
\begin{aligned}
& -F a \cos \beta+R \sin \varphi[a \sin \beta+b \sin (\alpha+\beta)]+ \\
& +R \cos \varphi[a \cos \beta+b \cos (\alpha+\beta)]=0 .
\end{aligned}
$$


From the equilibrium equation (3) we obtain an expression for finding the force $F$ of the spring's elasticity:

$$
F=\frac{R\{\sin \varphi[a \sin \beta+b \sin (\alpha+\beta)]+\cos \varphi[a \cos \beta+b \cos (\alpha+\beta)]\}}{a \cos \beta} .
$$

At a small angle of rotation $\beta$, the tension of the spring can be taken as equal:

$$
\Delta x=a \sin \beta .
$$

We find a connection between the magnitude of the tension of the spring and the deviation of the depth of the course:

$$
\Delta h=H_{1}-H_{2} .
$$

Based on the fact that

$$
H_{1}=a+b \cos \alpha,
$$

and

$$
H_{2}=a+b \cos (\alpha+\beta),
$$

we expect the deviation of the depth of the course:

$$
\Delta h=b[\cos \alpha-\cos (\alpha+\beta)] .
$$

Convert expression (9):

or

$$
\begin{gathered}
\Delta h=b[\cos \alpha-\cos \alpha \cos \beta+\sin \alpha \sin \beta], \\
\Delta h=b \cos \alpha(1-\cos \beta)+b \sin \alpha \sin \beta,
\end{gathered}
$$

остаточно знаходимо:

$$
\sin \beta=\frac{\Delta h-b \cos \alpha(1-\cos \beta)}{b \sin \alpha} .
$$

Substituting in formula (12) the value of the permissible deviation of depth $\Delta h$, and value $a, b$ and $\alpha$, it is possible to calculate the value of the permissible deviation angle.

Substituting (12) into formula (5), we obtain the magnitude of the maximum tension of the spring:

$$
\Delta x_{\max }=a \frac{\Delta h-b \cos \alpha(1-\cos \beta)}{b \sin \alpha} .
$$

Taking into account expressions (4) and (11), it is possible to calculate the stiffness of the spring $k$, in which the value of the deviation will be within the limits of agrotechnical tolerance:

$$
k=\frac{F}{\Delta x_{\max }} \text {. }
$$

The results of the calculations that were performed on a personal computer in accordance with the developed Mathcad program allowed the parameters of a new coil to be obtained, which show that, when the geometric parameters of the coil $a=120 \mathrm{~mm}, b=220 \mathrm{~mm}, \alpha=15^{\circ}, \beta=10^{\circ}$ the maximum tension of the spring should be $\Delta x_{\max }=14 \mathrm{~mm}$.

To overcome the force $R$ soil resistance on the basis of a coil, which is equal to $100 \mathrm{~N}$ for the angle of friction of the soil on the surface of the knife $\varphi=45^{\circ}$, according to the calculations according to formula (3), the spring must withstand force $F=258 \mathrm{H}$. Consequently, the stiffness of the spring of formula (14) should be equal $k=\frac{258}{0,014}=1,8 \cdot 10^{4} \mathrm{~N} \cdot \mathrm{m}^{-1}$.

Tests of combined two-disks and anchors, combined with new technical solutions, developed and manufactured at the NPC "Selta" NSC "IMESG" were conducted during program selection crops at the Institute of Irrigation Agriculture of the NAAS and other scientific institutions under the next program of experimental and agrotechnological research. The research was carried out in field crop rotation on dark chestnut medium-sandy soils. Millet in crop rotation was placed after winter wheat, the main treatment of 
soil was plowing to a depth of $20-22 \mathrm{~cm}$. Then, early spring harrowing was carried out, and at the optimal seeding time, pre-sowing cultivation was carried out at the recommended depth of seeding of 3 to $5 \mathrm{~cm}$. Seed was conducted immediately after cultivation. Agrotechnological research was carried out in field conditions with a sowing machine $\mathrm{CH}-16$ in an aggregate with a tractor T-25. The drill is equipped with 13 coils, of which: 7 pcs. - combined double-disk and anchor and 6 pcs. - Basic dual disks.

A comparative definition of the depth of the wrapping of millet seeds using different types of coulters and their impact on the uniformity of seedlings and development of plants has been carried out: a seed at a depth of $3 \mathrm{~cm}$ - the depth is observed, the seedlings are friendly (obtained on the 8th day of observations) when sown with the coulters of both structures ; sowing to a depth of $4 \mathrm{~cm}$ - the depth is observed, the stairs are friendly (received on the 9th-10th day of observation) in the sowing of the coulter of both structures; sowing to a depth of $5 \mathrm{~cm}$ - the depth is maintained, the stairs are friendly (received on the 12th day of observations) in the sowing of the coulter of both structures.

An estimation of the quality of the sowing (depth of wrapping, uniformity of seed distribution), depending on the speed of the sowing unit and the type of a comb in the aggregate with the tractor T-25 at speeds: at sowing at a speed of $7,2 \mathrm{~km} / \mathrm{h}$ - both types of combers ensure compliance with depth and uniformity distribution of seeds, as a result of the stairs received uniform and friendly; when sown at an elevated speed of $9.6 \mathrm{~km} / \mathrm{h}$, the anchor-disc type of the coulter ensured more accurate observance of the depth and uniformity of the seed distribution, resulting in a stair got uniform and friendly; at sowing at a speed of $14.5 \mathrm{~km} / \mathrm{h}$ - the observance of the depth and uniformity of the seed distribution ensured an anchor-disc type of coulter through seeding on a seedbed that created an anchor mounted on a spring suspension between the discs so that its deviation, regardless of seeding speed, maintains the depth of seeding within the limits of agrotechnological tolerance; A good effect was provided by the work of rolling rollers, which were equipped with two-disk anchor coils of a new design.

As the results of field experimental studies have shown, the speed of the sowing unit on the development of millet plants in the seeding of seeds with significant combines has no significant effect. The number of plants per linear meter is $21-22$ pieces, which in terms of one hectare makes $1,4-1,5$ million plants.

\section{Conclusions}

Theoretically, the parameters of the combined two-disk anchor coils are substantiated, prototypes have been made and field experiments and tests have been carried out. During the study, it was found that due to the addition of two-disk coulter with an additional pin-like part with a septum in such a way that a combined two-discus anchor coulter makes seeds on a solid seed bed, it eliminates the inertia of the removal of seeds beyond the limits of agrotechnological tolerance, including at higher seeding speeds. The equipment of the types of coulers and also the rolling hooks, which are installed with the ability to adjust their position relative to the coulter, create even greater advantages compared with other technical solutions. The economic effect of the introduction of this type of coulter on the breeding crops compared to sowing by standard two-disc coulters, depending on the seed sown, is from 300 to 900 UAH per 1 ha. Given the need to reduce the cost of material and technical resources and cash resources in crop cultivation technologies, it is advisable to equip small seedlings of type $\mathrm{CH}-16$ with new combined doubledisk anchor bits with rolling hooks.

\section{References}

1. Vasylchenko V. Factors that determine the quality of sowing/V. Vasilchenko, V. Opalko//Agronomist. — 2011. — № 1. - P. 186-189.

2. Vasilchenko V. Planters of grain seeders: features of structure and standard sizes/V. Vasilchenko,

O. Tkachenko, N. Vorontsova//Agronomist. - 2010. — № 4.- P. 140-146.

3. Gorobey V.P. Investigation of a two-disc combined coulter for sowing seed/V.P.. Gorobey, V.A. Luzin//Visn. agrar science. — 2010. — № 10. - P. 74-76.

4. Gorobey V.P. Determination of the stiffness of the spring of the combined double-disc anchor coil/V.P. Goroby//MESH. — 2015. — № 1. - P. 7-9. 
5. Dmitriev A.L. Modern agricultural machinery in the United States, England and in some other capitalist countries/A.L. Dmitriev//Mechanization of agriculture: coll. trans. and review. foreign. lit-ry; otv. Ed. A.V. Chumak. - Moscow: Izd-vo inostr. literary, 1955. - P. 5-66.

6. Levchuk M. Machines for sowing/M. Levchuk, V. Ziryanov //Farmer. - 2007. - № 4. - P. 3638.

7. Mechanization of field crop cultivation; Ed. A.N. Karpenko. - Moscow: Gos. i.e. literary, 1958. $533 \mathrm{p}$.

8. Peday N.P. Integrated mechanization of selection and experimental work in Russia/N.P. Peday//Mater. The 12th World. Conf. on the mechanization of field experiments. - Moscow: FGNU "Rosinformagrotekh", 2004. - P. 50-57.

9. Pogorily V. Each opener has its trump cards/V. Pogoryly, L. Shustik, S. Marinin//Grain. - 2011. № 2. - P. 90-92.

10. Saxton K.E. The main element of the seeder/K.E. Saxton//Grain. - 2007. — № 3. - P. 97-105.

11. Sysolin $P$. Provision of optimum grain crop sowing standards due to the quality of seeding/P. Sysolin//Technological and technological aspects of development and testing of new technology and technologies for agriculture in Ukraine. UkrNDIPVT. - Vol 6 (20). Kn. 1. - Doslidnitske, 2003. - P. 6872.

12. Theory, design and calculation of agricultural machines: a textbook for high schools of agricultural engineering/E.S. Bosoy, O.V. Vernyaev, I.I. Smirnov and others; under. Ed. E.S. Bosoy. - 2 nd ed., Rev. and enlaged. - M .: Mechanical engineering, 1978. - $568 \mathrm{p}$.

13. Shmat S.I. Possibilities of increasing the quality of two-disc coulter/S.I. Shmat, V.A. Reznichenko//Engineering in agricultural production, branch engineering, automation. - Vol. 17. KNTU. - 2006. - P. 79-81. 